\title{
PRIMARY CEREBRAL HYDATID CYSTS
}

\author{
BY \\ GILBERT PHLLIPS
}

Sydney, Australia

(RECEIVED FOR PUBLICATION, DECEMBER 1, 1947)

A review of primary cerebral hydatid disease in Australasia shows that twenty-nine cases of primary hydatid cyst of the brain have been recorded here during the last sixty years. The fact that such cysts are quite rare in countries where hydatid disease is relatively common has been stated previously by Dew (1934). In the opening paragraph of this article, Dew has clearly stated the basis for differentiating this type of cerebral cyst from secondary cerebral infestation : "As in the case of all organs, infestation of the brain with echinococcal disease may be either primary or secondary. In the former, the cysts are derived from hexacanth embryos, which, after passing through the liver and lungs, have been carried by the carotid arterial stream to the brain. Secondary cysts are metastatic, and result from the sowing by the blood stream of scolices, derived from a fertile primary simple cyst which has ruptured into the left side of the heart. It is obvious, although the fact has not been universally recognized, that the pathological and clinical aspects of these two types are very different ...."

The present article is concerned with an analysis of the twenty-nine recorded Australasian cases, which include five cases from the author's practice. Of the total twenty-nine cases, records of fourteen are contained in the Hydatid Registry of the Royal Australian College of Surgeons, five are recorded here, and the remainder are either referred to by Dew (1928) or have been published in early Australian medical periodicals.

An excellent review of the literature of intracranial hydatids has been published by Langmaid and Rogers (1940), and reference should be made to their article for an historical account of this condition.
Case Reports

Case 1. - A girl aged twelve years was referred to the author on June 25,1940 . This child had lived in the country all her life in a sheep-grazing district. She had been perfectly well until the past year, when she hado noticed attacks of giddiness which had become pro- $\Omega$ gressively more severe and more frequent and were accompanied by some alteration of visual acuity. In these attacks, objects seemed to rotate in a clockwise direction. In the past six months she began to experience left supra-orbital headache, which was worse on rising in the morning. Two months ago she noticed diplopia on lateral deviation to each side.

The only abnormal sign detected on examination of the nervous system was a complete congruous right homonomous hemianopia with macular sparing. There was no enlargement of the head, and radiographs of the skull were within normal limits. The girl was bright, alert, and intelligent, and co-operated well during examination. There was no papillodema. The cerebrospinal fluid pressure was $350 \mathrm{~mm}$., chlorides $710 \mathrm{mg}$. per $100 \mathrm{c.cm}$.; there was no increase in globulin, and the protein content was $20 \mathrm{mg}$. per $100 \mathrm{c.cm}$. The Casoni reaction and complement fixation test for hydatid were both negative. There was no relative eosinophil leucocytosis. A pre-operative diagnosis of left occipital tumour was made, and a left occipital craniotomy performed. The exposed brain appeared bluish, and an incision into the left occipital region revealed the greyish wash-leather wall of a hydatid cyst. The cortical incision was extended, and the whole cyst removed intact. After removal, examination of the cystic fluid did not reveal any scolices or hooklets. The wound was closed in layers without drainage; convalescence was uneventful and the patient was discharged from hospital perfectly well three weeks after operation. She has remained quite well, and has been working for some years as a telephonist. 
Case 2.-A boy aged ten years was referred by Dr. Holt, Canberra, Australian Capital Territory, on Nov. 9, 1940. He had been perfectly well until nine months previously, when he had a series of generalized convulsions, since when he developed tremor in the left hand, generalized headaches, and occasional early morning vomiting. Apart from these symptoms he appeared quite well. He could swim and ride a bicycle, and was good at his schoolwork. His visual acuity was $6 / 6$ in each eye, there was no papillodema, but perimetric examination showed a left congruous homonomous hemianopia with macular sparing. There was no other disturbance of the cranial nerves or of the motor or sensory systems.

Plain radiographs of the skull showed some increase in convolutional markings, but were otherwise within normal limits. The cerebrospinal fluid was clear, under a pressure of $300 \mathrm{~mm}$. There was no increase in cells or globulin, the total protein was $10 \mathrm{mg}$. per $100 \mathrm{c.cm}$., and the chlorides were $730 \mathrm{mg}$. per $100 \mathrm{c.cm}$. The Casoni reaction and complement fixation test for hydatid were negative. A provisional diagnosis of right occipital tumour was made, and a ventriculogram performed by the occipital route. On the right side, at $1 \mathrm{~cm}$. below the dura, yellowish fluid was aspirated. In view of the colour of the fluid, it was considered that the lesion was a cystic glioma, and a bone flap was reflected. An incision was made into the right occipital cortex, and the cyst opened and then found to contain $12 \mathrm{oz}$. of yellowish fluid and many daughter cysts. Microscopic examination of the fluid showed profuse scolices. Failure to recognize the fact that hydatid fluid might be yellowish had meant that the operation field was now contaminated, and at this stage it was too late to disinfest with formalin solution. The patient made an uneventful recovery, and was discharged from hospital three weeks after operation. He remained well for seven months, and then commenced to suffer from severe generalized headache, following which he became progressively stuporose and died in coma eight months after the. operation. Post-mortem examination was carried out twenty-five hours after death. There were no hydatid cysts present in the lungs, heart, or liver. During removal of the brain a large number of hydatid daughter cysts, each about two $\mathrm{cm}$. in diameter, escaped from the right cerebral hemisphere. These cysts were found mainly in the right temporal lobe, but were also present in the adjacent parts of the right parietal and occipital lobes. Death had been due to medullary compression following uncal herniation.

Case 3.-A boy aged eleven years was referred to the Neurosurgical Department of the Royal Prince Alfred Hospital by Dr. Glanville Rawle of Lismore on June 5, 1941. For two years he had shown slowly progressive mental deterioration, and during the past twelve months he had begun to suffer from generalized headaches. In the past two months his visual acuity had gradually diminished.

The child had a large head, a fatuous smile, and monosyllabic, monotonous speech. His mentality was well below the average. The bitemporal diameter of the head was $17 \mathrm{~cm}$., the occipito-frontal diameter $21.5 \mathrm{~cm}$. There appeared to be general widening of the sutures, especially marked over the left fronto-parietal region. Both eyes showed some degree of proptosis, the left being more marked than the right. There was no anosmia, the appearance of the optic discs suggested early papillodema and the remaining cranial nerves were all intact. There was no disturbance of the motor system, his co-ordination was intact, and all the tendon reflexes were present, slightly increased, and equal on the two sides. The abdominal reflexes were present.

Owing to mental deterioration, he was a poor witness for subjective tests, but no disturbance of the sensory system could be detected, and the visual fields appeared full on confrontation. Radiographs of the skull showed a very large head with widely separated sutures, a " ballooned" sella turcica, and marked thinning of both frontal bones. The cerebrospinal fluid pressure was $300 \mathrm{~mm}$., the fluid clear; there was no increase in globulin, the total protein was less than $20 \mathrm{mg}$. per $100 \mathrm{c.cm}$., and the Lange gold curve 0001100000 ; there was no increase in cells, and the Wassermann reaction was negative. The Casoni reaction and complement fixation test for hydatid were positive, and the eosinophil differential count 5 per cent.

A ventriculogram performed by the occipital route showed considerable shift of a dilated ventricular system to the right side. The left frontal horn did not fill. A diagnosis of left frontal hydatid cyst was made. Craniotomy was performed by the left frontal approach, and the exposed cortex appeared extremely thin and presented a blue-grey appearance. The thin shell of cortex (one or two $\mathrm{mm}$. thick) was removed to uncover the wall of the cyst, and the cyst was needled and a large amount of cloudy fluid gushed out. Eight c.cm. of 10 per cent. formalin was run into the cyst and allowed to remain in situ for seven minutes. The contents of the cyst were then completely evacuated, and the cyst was opened and its inner wall painted with metaphen. The wall was then delivered in one piece, and could be stripped away with ease from the adjacent cerebral surface. The volume of the evacuated fluid measured $20 \mathrm{oz}$.

The patient's condition was excellent throughout the operation, and his convalescence was uneventful. He was allowed out of bed ten days after the operation. Post-operative radiographs showed that the cavity left by removal of the cyst was filled with air, which gradually disappeared over the succeeding week, apparently as the result of expansion of the left cerebral hemisphere.

This patient has been seen from time to time during the past six years, and is at present quite well and has had no recurrence of his previous symptoms.

Case 4.-A boy aged three years and four months had lived in Harden, New South Wales, since birth. He lived on a farm where there were four dogs used in relation to sheep grazing. He was referred to the care of Dr. J. S. Davis at the Royal Alexandra Hospital with a history that he had been limping on the left leg for six to eight weeks and was disinclined to use the left arm. There had been no complaint of headache, no vomiting 
and no obvious change in the visual acuity. The child's head was enlarged (circumference 22 inches), and the vault appeared to be more prominent in the right parietal region. Well marked papillodema was present in each fundus, but there appeared to be no contour defect in the visual fields an confrontation. The left abdominal refiexes and the left knee jerk were absent. Plantar responses were flexor on both sides. The Casoni reaction was negative, and there was no increase in eosinophil leucocytes.

A provisional diagnosis of a right hemisphere lesion was made, and a ventriculogram was performed. Clear fluid was withdrawn from the right side at a depth of $1.5 \mathrm{~cm}$., and it was supposed that a dilated ventricle had been tapped. Air was introduced into the supposed ventricle and the head radiographed. At this stage the author was asked to see the case in consultation, and it was decided that the air had filled a cystic cavity which was probably a cerebral hydatid. A right parietal fiap was then reflected, and, after incision of the dura, the typical wash-leather wall of a hydatid cyst was seen presenting on the surface. After the field had been packed off, $5 \mathrm{c.cm}$. of formalin solution were injected into the cyst, after removal of a similar volume of the cystic fluid. Five minutes later the contents of the cyst were aspirated and the cyst wall delivered intact. The next morning the child had repeated generalized convulsions, and these were controlled with a basal dose of " avertin." Scolices were found in the cyst wall after removal. The child was discharged from hospital five weeks after operation.

Case 5.-A woman aged twenty-three years was referred to the author by Dr. John McGeorge on July 19, 1943, with a provisional diagnosis of cerebral tumour. This patient had never lived in the country, although she had had a good deal to do with dogs, both in her home and elsewhere. She had been perfectly well until twelve months previously, when she began to get occasional generalized headaches. Two to three months before she was seen they had become more severe and were accompanied by early morning vomiting. On three occasions in the early morning she became giddy and had lost consciousness for a period of three to five minutes. There was nothing relevant in the family history, apart from the death of a sister a year previously with tuberculosis. She was married but had no children.

She was a rather masculine type, but was mentally bright and co-operated well during examination. Bilateral papillœdema was present; the left pupil was larger than the right; both reacted well to light and accommodation. There was coarse nystagmus on looking to the left. The remaining cranial nerves were intact, there was some past-pointing to the left with the right hand and some tremor of the left arm. Her tendon refiexes were present and equal on the two sides. The plantar responses were absent on each side. There was no dysdiadokokinesia and no disturbance of gait. Visual field examination showed a left upper congruous quadrantopsia with macular sparing. A plain radiograph of the skull showed considerable enlargement of the pituitary fossa with destruction of the posterior clinoid processes and dorsum sellæ. The cerebrospinal fluid pressure was $420 \mathrm{~mm}$., the globulin was not increased, and total protein was $20 \mathrm{mg}$. per $100 \mathrm{c.cm}$. The Casoni reaction and complement fixation test for hydatid were negative.

In view of the visual field defect, it was decided that this patient had a tumour in the right temporal lobe, and a ventriculogram was performed and air introduced into the large cyst in this position. The cyst was very large, extending almost to the cortical surface and medially to the midline. Its anterior-posterior extent was approximately $6 \mathrm{~cm}$. In view of these appearances, it was suspected that it was hydatid in origin, and a right temporoparietal flap was turned down. The cyst was again tapped, and sterilized by the introduction of $10 \mathrm{c.cm}$. of 5 per cent. formalin, which was allowed to remain in situ for ten minutes. The contents of the cyst were then evacuated. The cyst wall was very thin and friable, and broke up with handling. It could not be removed, and it was then decided to close the wound. The cystic fluid did not show any hooklets or scolices.

Two weeks after the operation the patient developed gross cdema of the face and a bilateral lower motor facial palsy, which subsided rapidly in four days following massive vitamin $B_{1}$ injections. She was discharged quite well three weeks after operation. Four months after operation she was re-admitted to hospital and an encephalogram performed, which showed a normal ventricular system. She has been observed at intervals during the past four years, and has had no recurrence of symptoms. She was again admitted to hospital in March, 1947, for a further encephalogram, and again the ventricular system was seen to be normal.

\section{General Considerations}

The main features of the preceding five cases reported from the author's practice are abstracted and are included in the Table with the previous twenty-four cases in the Australasian literature. Primary cerebral hydatid cysts are single and are due to the passage of a hexacanth embryo through the hepatic and pulmonary circulation with ultimate embedding in a cortical capillary. According to Dew they are at least seven times more common in children than in adults, for he says that cerebral hydatid cysts form 4.9 per cent. of all organ infestations in children, and 0.7 per cent. of all organ infestations in adults. The relative frequency in children in this series appears very much higher, since only two cases out of twenty-nine were above seventeen years of age. The hemisphere involyed would appear to be quite fortuitous. Dew has suggested, though he qualifies his statement by saying that there are not sufficient statistics for adequate deductions, that the left carotid would receive the larger percentage, and that primary cysts should be more frequent in the left cerebral hemisphere. In this series, of the twenty-eight cysts in the cerebral hemispheres, fifteen were found 
on the left side and thirteen on the right. The one cerebellar cyst reported was on the left side. It would seem that from these figures no deduction may be drawn, except that relative infestation of the two sides is about equal. It is remarkable that the nine New Zealand cases all occurred in the right hemisphere, and sixteen of the twenty Australian cases occurred on the left side.

Dew (1928) has already pointed out that the parietal lobe is the most frequent site for primary cerebral hydatid cyst; the present series shows the following distribution :

$\begin{array}{lllllr}\text { Frontal } & . & \ldots & \ldots & \ldots & 2 \\ \text { Parietal } & . & \ldots & . & \ldots & 14 \\ \text { Parieto-occipital } & . & . & \ldots & 5 \\ \text { Temporal } & . & \ldots & \ldots & \ldots & 2 \\ \text { Occipital } & . . & \ldots & \ldots & \ldots & 5 \\ \text { Cerebellar } & \ldots & . . & \ldots & 1 \\ & & & & & 29\end{array}$

It should be remembered, however, that these cysts are often so large that they overlap the boundaries of cerebral lobes, and may not infrequently occupy the parieto-temporo-occipital region of one half of the cranial cavity. It is more useful, therefore, to compare a pre-rolandic with a post-rolandic distribution. By far the greater number of cysts are found in the vascular field of the middle cerebral artery, and consequently come to lie mainly behind the fissure of Rolando. This might be expected from the relative size of the middle cerebral artery compared with the smaller lumen of the anterior cerebral and posterior communicating vessels, and from the nature of the origin of the middle cerebral artery from the internal carotid.

In this series the hemisphere cysts were distributed as. follows :

$\begin{array}{llllr}\text { Pre-rolandic } & \ldots & \ldots & \ldots & 2 \\ \text { Post-rolandic } & \ldots & \ldots & \ldots & 26\end{array}$

As the cyst grows, it displaces the cerebral substance and adhesions do not tend to form between the brain surface and the adventitia of the cyst. For this reason, the whole cyst wall may usually be delivered with care, after sterilization and partial evacuation, by gentle traction with widetipped forceps, such as the sponge-carrying type. The rate of growth of hydatid cysts of the brain is probably quicker in the brain than in other organs, at least in the early stages before an increase in intracranial pressure has taken place. In Case 3 reported above, multiple cysts were found at autopsy to have reached a maximum diameter of $2.5 \mathrm{~cm}$. eight months after an operation for removal of the primary cyst during which the operation field was sown with scolices. It may be concluded, therefore, that a primary cerebral hydatid cyst of $2.5 \mathrm{~cm}$. diameter had been sown in the brain approximately six to twelve months previously.

The cyst is usually perfectly circular in shape, and, if it does not actually present on the surface, it will be found to be covered by not more than a few millimetres of compressed cortex. In some cases actual erosion of the overlying cranial vault may be found, and this fact should be remembered in studying skull radiographs. In one case the cyst had eroded through the calvarium and was presenting beneath the scalp. The wall of the cyst has a characteristic anæmic wash-leather appearance, and is usually about 2 to $3 \mathrm{~mm}$. thick. Its laminated character is certain evidence of hydatid pathology. The cyst contents may or may not include scolices, hooklets, or daughter cysts. The cyst is often sterile, and daughter-cyst formation is infrequent. The hydatid fluid in these cysts is usually perfectly clear, and may at operation be indistinguishable from ventricular fluid. Occasionally it may be cloudy or even yellowish in colour. The saline content may or may not be increased.

\section{Diagnosis}

Even in a country where hydatid disease is relatively common, the pre-operative diagnosis of a primary cerebral hydatid is uncommon. This might be expected in the first instance from the relative infrequency of primary cerebral infestation, and precise diagnosis is made difficult by the fact that many patients do not exhibit any specific local or general evidence of hydatid infestation. In only one case (25) in this series were the specific hydatid reactions positive, and it is not surprising that in the twenty-nine cases a pre-operative diagnosis of primary cerebral hydatid cyst was made in only eight cases.

The symptomatology usually implies the presence only of some space-occupying lesion causing increased intracranial pressure, and, even after exhaustive investigation which may confirm the presence and position of the lesion, its nature may not be proved until a craniotomy has been performed and the wall of the cyst exposed. However, the general clinical picture is sufficiently uniform for suspicion of a hydatid lesion to be aroused whenever such a picture is seen in a child referred from a sheep-grazing district. If one is not aware of this possibility beforehand, the intracranial field may be sprayed with scolices through the performance of a ventriculogram or an improperly planned operation (Case 2).

The average age at which symptoms present themselves is important in diagnosis. In this series, 


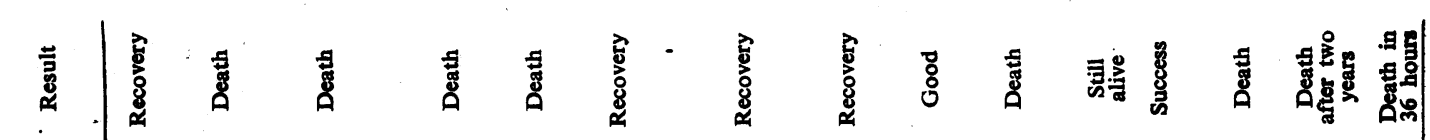

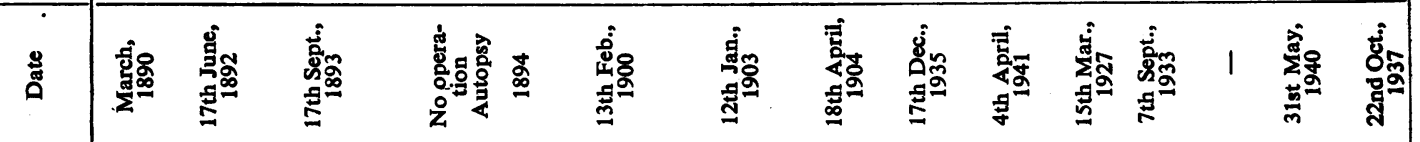

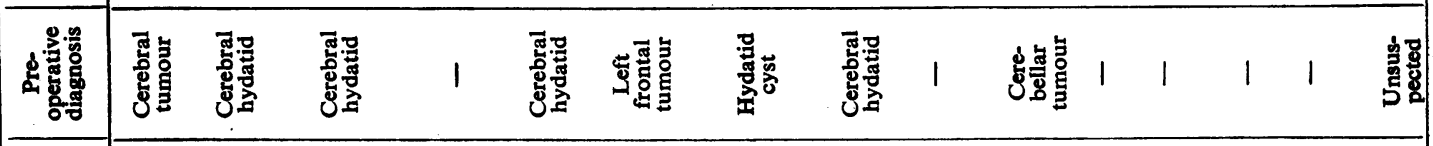

\begin{tabular}{llllllllllllllll}
\hline & 1 & 1 & 1 & 1 & 1 & 1 & 1 & 1 & 1 & 1 & 1 & 1 & 1 & 1
\end{tabular}

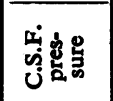

त्?

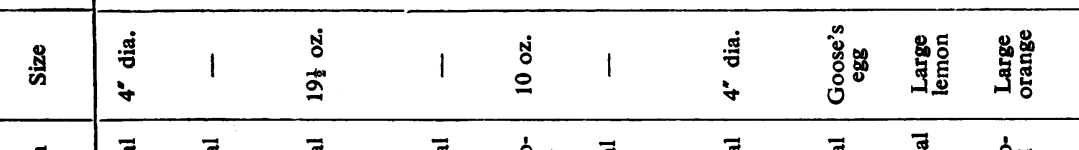

\begin{tabular}{|c|c|c|c|c|c|c|c|c|c|c|c|c|c|c|}
\hline 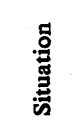 & 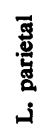 & 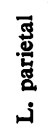 & 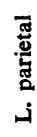 & 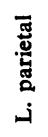 & 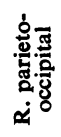 & 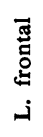 & 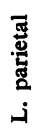 & 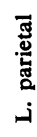 & 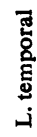 & 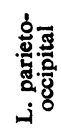 & 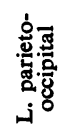 & 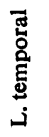 & 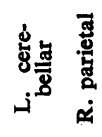 & \\
\hline
\end{tabular}

量

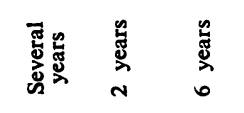

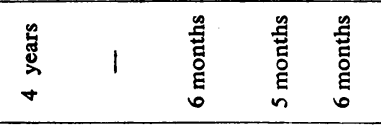

龸

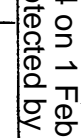

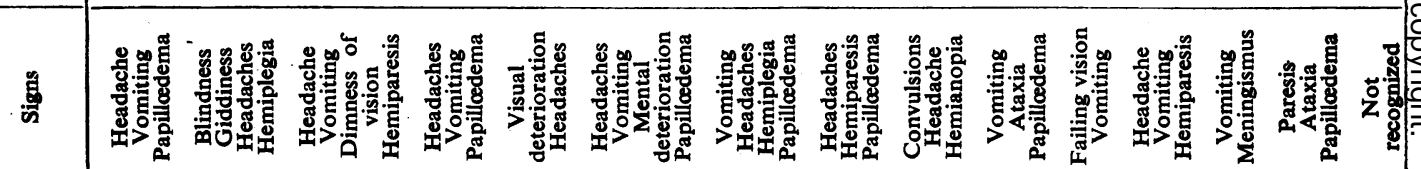

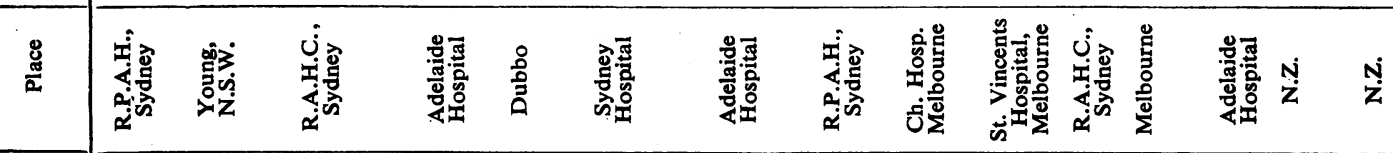

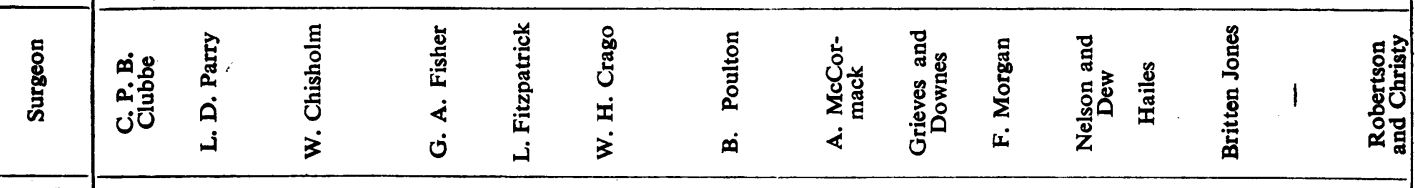

\begin{tabular}{|c|c|c|c|c|c|c|c|c|c|c|c|c|c|c|c|}
\hline 造 & $\underline{0}$ & $a$ & $\simeq$ & $\Xi$ & $a$ & $m$ & $=$ & 0 & 은 & $m$ & $\simeq$ & $\exists$ & 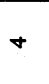 & $\infty$ & $a$ \\
\hline
\end{tabular}

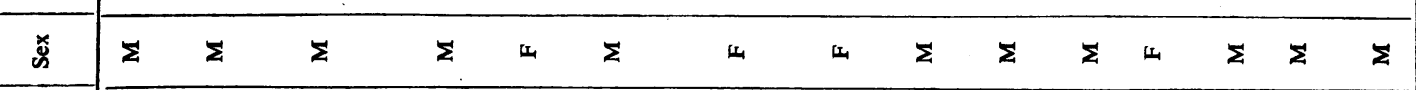

焉

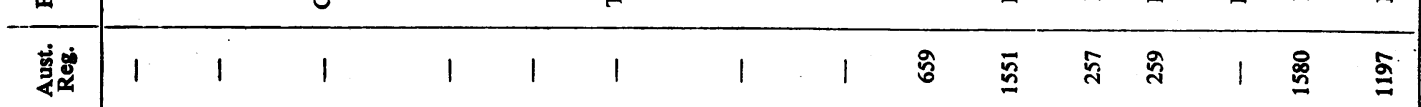

岁: 


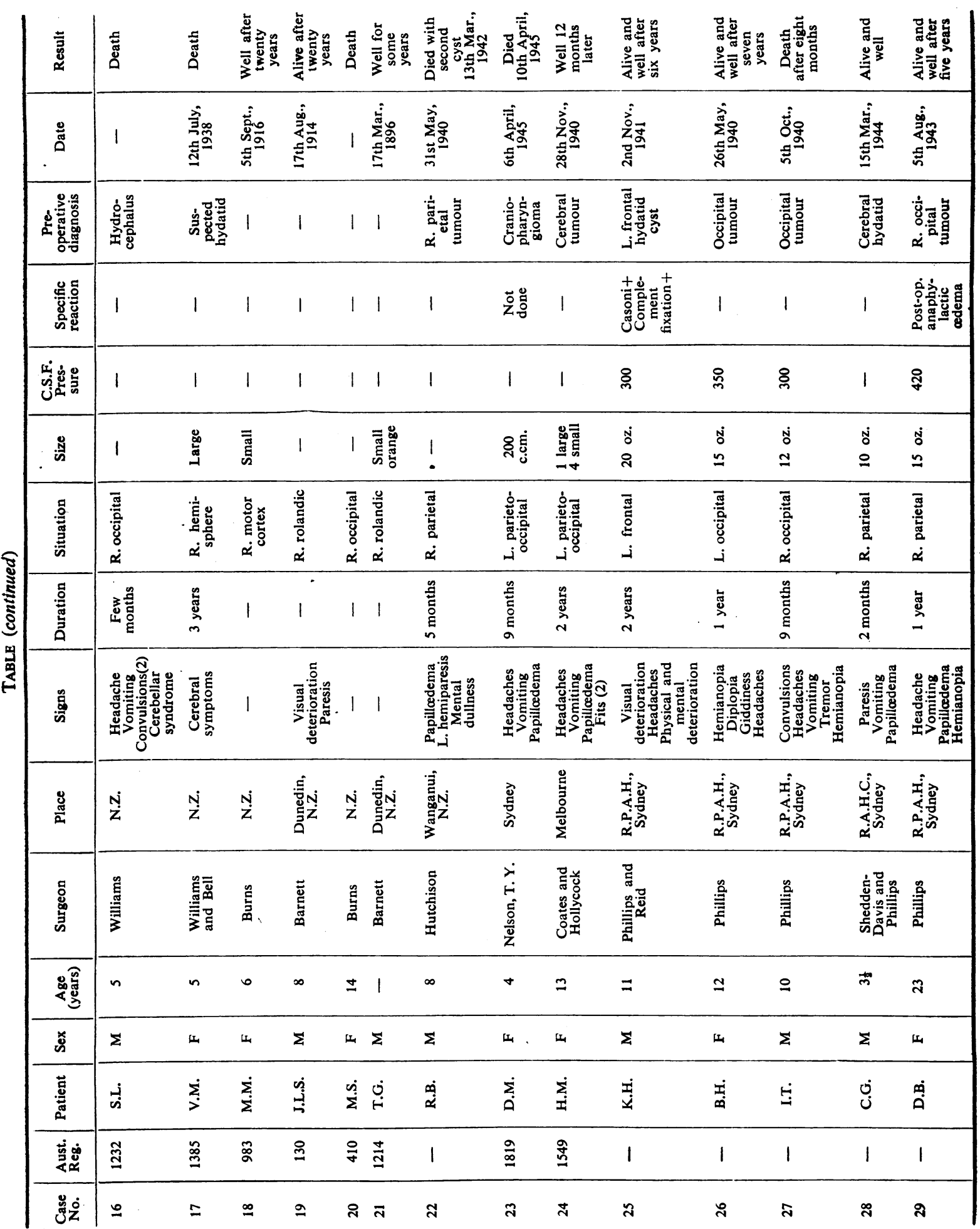


the extremes range from three to forty-four years. However, eighteen cases occurred in the decade from seven to seventeen. The average age at operation lies between nine and ten years, premonitory symptoms usually arising one or two years previously. Even in Case 10 (aged three) the symptoms had been present for six months, and, as the cyst was described as being the size of a large orange, it had probably been sown in this patient's brain at about the age of eighteen months. There is a preponderant infestation of males as against females in a ratio of eighteen cases to eleven cases in this series.

The cardinal symptoms in practically all the cases are those due to increased intracranial pressure, particularly vomiting and headache : one, or more commonly both, of these symptoms were present in every. one of the cases. Their character is identical with the same symptoms produced by intracranial neoplasm. The headache is usually generalized and appears on rising in the morning. Vomiting, which also tends to occur in the early morning, is projectile, is not preceded by nausea, and may relieve the headache. There may be, and usually are, no other symptoms, at least in the early stages. Visual deterioration, which is either due to optic atrophy following papillødema or to hemianopia, is a fairly constant late symptom. Unilateral weakness is common as a late symptom, and may be associated with a history of convulsions. Less commonly, as a late manifestation, cerebellar symptoms may be present, as they were in two of the author's cases, and may constitute a false localizing sign suggesting the presence of a lesion in the posterior fossa (see also Case 10).

The signs of primary cerebral hydatid cyst relate chiefly to the increase in intracranial pressure, and it is rare that the patient is proved in the first place to be the host of a hydatid cyst in another situation. Autopsy in Case 27 did not reveal evidence of hydatid infestation anywhere else in the body, and a number of previous autopsy reports in patients who have died from primary hydatid cyst of the brain have rarely shown the presence of hepatic or pulmonary lesions. Those who live after operation have usually done so for many years, a fact which indicates that the cerebral cyst was single, both in the brain and the body. In most cases where the Casoni and hydatid precipitin reactions were examined they were found to be negative. In Case 25 they were both positive. The non-specific signs include papillœdema, a large head, not infrequently a cracked-pot sound on percussion, and radiographs which show convolutional thinning, separation of sutures, or erosion of the sella turcica. Local thinning of the calvarial vault may occasionally be seen. Calcification of the pineal gland is unusual in children, so that lateral shift may not be detected radiographically. Calcification has not been recorded in a primary cerebral hydatid cyst. Hemianopia may be a useful sign, and was found in three of the author's cases before operation. From the situation of the cyst in Case 28 it may be deduced that hemianopia was probably present there as well, but the patient was too young for accurate subjective examination. Signs of pyramidal disturbance may be seen in convulsions or paresis ; tremor, ataxia and nystagmus occurred in a number of cases due to involvement of the extrapyramidal circuit from cortex to cerebellum.

Individual symptoms and signs may now be examined in some detail.

Headache, Vomiting, and Papilloedema.-This group of pressure symptoms may conveniently be tåken together. As one might expect in a group of people whose average age is at the beginning of the second decade in life, vomiting was the commonest sign in the pressure group. Characteristically projectile, it was typical in other respects in its appearance early after rising in the morning, and in the relief of the morning headache which succeeded vomiting. Headache was nearly as common, was generalized, and had the characteristic early morning incidence of a pressure headache. Papillodema was less common, being recorded in eleven of the twenty-nine cases, the low incidence no doubt being due to the separation of sutures which may occur in this age group. The pressure group of symptoms is, however, most important, as one or more of the three may be the only evidence of a primary cerebral hydatid cyst in a child, and even exhaustive investigation may not reveal any other significant sign.

Visual Disturbances.-In the records of the early cases, visual disturbance is usually stated simply as visual deterioration, and in only one case (Case 9) had hemianopia been reported, though it was observed in three of the author's five cases. There is little doubt that in these children, particularly in the late stages, visual deterioration will occur as the result of secondary optic atrophy following papillœdema. However, as stated previously; with a focal distribution of thirteen to one in favour of a post-rolandic site, and with lesions which usually contain from ten to twenty ounces of fluid, an early high incidence of hemianopia is to be expected. As the defect will be due to interference with the supra-geniculate pathway, macular sparing should 
be typical and central vision therefore is unlikely to be affected. However, the child may be aware of some change in visual capacity, which he is probably unable to assess or describe, and which may therefore be exhibited wholly by errors in school work and a tendency to bump into objects on the side of the visual field defect. There is probably little doubt that in the majority of primary hydatid cysts in the cerebral hemispheres a careful perimetric examination will disclose a congruous hemianopia with macular sparing, and if this is found in association with signs of increased pressure a diagnosis of primary cerebral hydatid cyst becomes more likely. At this age, and in this situation, other lesions become even less common than primary cerebral hydatid cyst.

Pyramidal Disturbances.-Motor disorders may take the form of paresis, tremor, or convulsions. Paresis is by far the most common, having occurred in twelve cases in this group, in most instances involving the contralateral arm and leg equally. In a few cases tremor of the paretic limb was the first sign of pyramidal defect. It is unusual that in this age group convulsions were not more commonly seen, having been reported in only one of the author's cases and in three cases previously. Unfortunately, electroencephalographic studies are not available in any of this material.

Mental Deterioration.-In seven cases, some form of mental deterioration has been observed, and it is notable that this was present both in the frontal cysts and in the post-rolandic cases ; it was usually seen in a patient in whom symptoms had been present for a long time or in whom the cyst was a large one.

Cerebellar Signs.- These include ataxia, nystagmus, and intention tremor, and they may be false localizing signs. Mild defects suggestive of cerebellar dysfunction have been recorded in a number of cases, and it has been suggested that they may be due to pressure on the ipsilateral cerebellar hemisphere from the large cystic collection lying above the tentorium. That this is not the explanation may be deduced from the fact that the apparent cerebellar disturbance is on the opposite side to the cyst in the cerebral hemisphere, and it is probably interference with the temporoand occipito-pontine fibres relaying into the neocerebellum that produces disturbance of coordination and decomposition of movement, particularly in the upper extremity on the opposite side of the body.

\section{Surgical Treatment}

The surgical removal of primary hydatid cyst of the brain is a simple procedure and offers few technical difficulties. The skull is usually thin, and a large bone flap may be reflected in most cases without any blood loss, as the bone itself and the intracranial field are both relatively avascular. In one of my cases the bone was so thin that the flap could be cut down with strong scissors. The dura having been reflected, the wall of the cyst may be found to present on the surface, or may be covered with avascular cortex which is not more than a few millimetres thick and which may therefore be split in a linear fashion to expose the firm anæmic wash-leather membrane whose appearance is sufficiently characteristic to establish the diagnosis in most cases. When the cyst is recognized, the exposed cortex should be covered by overlapping wet lintine strips covering the whole area with the exception of a small point on the cyst wall.

Five c.cm. of 10 per cent. formalin are now placed in a $10 \mathrm{c.cm}$. syringe. A fine needle is attached to the syringe, and, after insertion through the cyst wall, $5 \mathrm{c.cm}$. of cyst fluid is withdrawn slowly into the syringe. The contents of the syringe are now injected, without pressure, into the cavity of the cyst. It is wise to have a loose piston in the syringe, and to allow the formalin-cyst fluid mixture to run back by gravity. Two or three c.cm. may remain in the barrel. The needle may then be withdrawn and a lintine patty placed over the point of its insertion. The formalin should be allowed to act in the cyst for ten minutes before any other step is taken.

To remove the formalin, a rubber-type syphon may be attached to a needle reinserted into the cyst wall and the contents syphoned off into an open vessel held away from the operation field. During this procedure the cyst wall tends to collapse away from the surrounding brain tissue and cerebral pulsations of large amplitude begin. Through a second needle inserted into the cyst, further irrigation of the cyst contents may be carried out through the syphon. It is difficult to remove all the fluid within the cyst, and an opening should be made into the wall at this stage at the highest point presenting. The remaining fluid may then be aspirated with a sucker under direct vision. The interior of the wall may then be painted lightly with formalin or metaphen, if the aspirated fluid shows the presence of hooklets or scolices.

The cyst wall may now be withdrawn by gentle traction, care being taken to see that no large veins have become trapped in the collapsed folds of the 
52

GILBERT PHILLIPS

cyst. In nearly every case, gentle steady traction of the wall by a pair of sponge-holding forceps in any damage to the surrounding brain tissue. At this stage the cavity may be lightly filled with saline and the field closed in the usual manner.

The anesthetic of choice is rectal avertin, which not only reduces the intracranial pressure during operation, but keeps the patient at rest for some hours after, during the early expansion of brain tissue to fill the cavity, which may have contained as much as a pint of fluid. This expansion is usually complete, and postoperative encephalograms have revealed a normal ventricular system in several cases.

The chief difficulty lies in the approach to operaton. If hydatid cyst is expected, air studies should not be carried out. Localization may usually be made from the clinical picture, and a bone flap should then be reflected and the cyst wall inspected. The fundamental principle involved is to avoid contamination of the field by scolices, either at operation or by such procedures as ventriculography, or by an attempt to aspirate the cyst through a single burr hole.

My thanks are due to Professor Harold Dew and to Sir Hugh Cairns
of this paper.

Dew, H. R. (1934). Surg. Gynec. Obstet., 59, 321. Dew, H. R. (1934). Surg. Gynec. Obstet., 59, 321.
Publishing Co. Sydney. p. 353.
(1928). Hydatid : Brain, 63, 184.

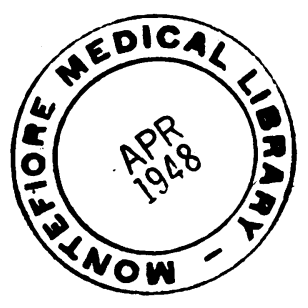

